\title{
Effects of melatonin on the nitric oxide treated retina
}

\section{A W Siu, G G Ortiz, G Benitez-King, C H To, R J Reiter}

Br J Ophthalmol 2004;88:1078-1081. doi: 10.1136/bjo.2003.037879

\begin{abstract}
Aims: Nitric oxide (NO) is a free radical which reportedly causes damage to living cells. This study evaluated the damaging effect of $\mathrm{NO}$ and the protection of melatonin on the retina in vivo.

Methods: Female Wistar rats $(230-250 \mathrm{~g})$ received two intraperitoneal injections of either melatonin $(5 \mathrm{mg} / \mathrm{kg})$ or vehicle alone. After general anaesthesia, the animals received $1 \mu \mathrm{l}$ intravitreal injections of $0.9 \%$ saline and $1 \mathrm{mM}$ sodium nitroprusside (SNP) into the right eye and the left eye, respectively. The animals were divided into two groups and then sacrificed after 24 hours (day 1) and 96 hours (day 4). The mean inner retinal layer thickness (mIRLT), the number of retinas expressing hyperchromatic (HC) nuclei in the inner nuclear layer (INL) and the apoptotic ganglion cell detection were compared.

Results: After 1 day, SNP significantly increased the mIRLT by $45 \%(p=0.004)$, initiated more INL nuclear HC expression $(p=0.01)$ and apoptotic nuclei $(p<0.05)$ compared with the control eyes. Injection of melatonin ameliorated these changes. On day 4, SNP demonstrated similar effects in all parameters on the retina. After the injection of melatonin, both INL HC expression and apoptotic ganglion nuclei in the SNP treated eyes were similar to the controls but the mIRLT was significantly greater than in controls $(p=0.006)$.

Conclusion: Uncontrolled NO elevation caused morphological and nuclear changes in the retina. Melatonin significantly suppressed the NO induced increase in mIRLT, INL HC expression, and apoptotic ganglion cells on day 1, but not after day 4. Melatonin may have a protective role in the NO elevated retina.
\end{abstract}

l traocular pressure elevation, a risk factor of glaucoma results in an increased nitric oxide (NO) level in the retina. $\mathrm{NO}$ is a nitrovasodilator and this upregulation may have a beneficial role by increasing the retinal blood circulation in reaction to the increased pressure. However, NO combines easily with the superoxide anion radical to produce the toxic peroxynitrite $\left(\mathrm{ONOO}^{-}\right)$anion. ${ }^{2} \mathrm{ONOO}^{-}$is a highly reactive oxidative species which may challenge normal enzymatic function, ${ }^{3}$ alter membrane fluidity, ${ }^{4}$ and disrupt ionic transport. ${ }^{5}$ The retina has a high content of polyunsaturated fatty acid and it is susceptible to free radical toxicity. Hypothetically, an increase in intraocular free radical, such as NO, would pose an oxidative stress to the retina. However, the question as to whether excessive, uncontrolled NO production causes retinal degeneration remains to be elucidated.

Melatonin is an indoleamine produced in the pineal gland and the retina. In the retina, melatonin receptors are clustered on the photoreceptors, inner segments, and ganglion cells. ${ }^{7}$ Melatonin is known to regulate the circadian rhythms ${ }^{8}$ but the production of melatonin in the retina indicates a local non-endocrine role. Increasing evidence suggests that melatonin is a potent antioxidant ${ }^{9}$ and it protects the central nervous system from oxidative damage. ${ }^{10}$ Melatonin also suppressed NO induced retinal lipid peroxidation $^{11}$ and ischaemic retinal neuron death ${ }^{12}$ in vitro. However, little information is available in the literature with regard to the antioxidative role of melatonin on the NO treated retina in vivo. This study aimed to investigate the effects of excessive NO on the retina shortly after the treatment and the role of melatonin in NO treated retina.

\section{MATERIALS AND METHODS}

Sodium nitroprusside (SNP) was purchased from SigmaAldrich Inc (St Louis, MO, USA). Melatonin was obtained from Helsinn Co (Biasca, Switzerland). Female Wistar rats (230-250 g) were ordered from the animal unit of the Centro de Investigación Biomédica de Occidente (IMSS), Guadalajara, Jalisco, Mexico. Twenty animals were equally divided into two groups and each animal received two consecutive (30 minutes apart) intraperitoneal injections $(0.4 \mathrm{ml})$ of either melatonin $(5 \mathrm{mg} / \mathrm{kg})$ or alcoholic saline only. Another three animals did not receive any treatment and served as baseline controls.

Thirty minutes after the second injection, the animals were anaesthetised (Droperidol and Ketamine mixture). Five minutes later, each animal received an intravitreal injection of $0.9 \%$ saline in the right eye and $1 \mathrm{mM}$ SNP in the left eye ( $1 \mu \mathrm{l}$ in all cases). The injections were made at the temporal sclera posterior to the limbal region using a Hamilton syringe. After the injections, the rats were returned to a 12L:12D light controlled unit. Food and water were available ad libitum. Half of the rats were killed on day 1 (after 24 hours) by decapitation while the remainders were killed on day 4 (after 96 hours). The procedure was reviewed by the institutional subcommittee of animal research and it was in accordance with the proposed guidelines of Association for Research in Vision and Ophthalmology for the use of animals in ophthalmic and vision research.

The retinas were collected from the eyecups. The tissues were fixed in $0.1 \mathrm{M}$ phosphate buffered paraformaldehyde $(4 \%)$ and glutaraldehyde (1\%) solution overnight at $\mathrm{pH}$ 7.4. The samples were post-fixed in osmium tetroxide $(1 \%)$ and dehydrated in graded concentrations of alcohol. Thin radial sections $(2 \mu \mathrm{m})$ embedded in epoxy resin were obtained at approximately $1 \mathrm{~mm}$ superior to the optic disc. Only sections with the outer nuclear layer not exceeding 11 nuclei in a column were accepted. The retinal sections were mounted on microscopic slides and stained with toluidine blue.

\section{Morphometry}

The sections were studied under a light microscope (Olympus Vanox-1). The inner retinal layer thickness (IRLT) was determined using a computerised image analysis program (Image-pro plus, v 1.3). The IRLT was taken as the distance

Abbreviations: HC, hyperchromatic; INL, inner nuclear layer; IRLT, inner retinal layer thickness; ONOO ${ }^{-}$, peroxynitrite anion; SNP, sodium nitroprusside 
from the outer margin of inner nuclear layer (INL) to the inner limiting membrane of the ganglion cells layer. An average of three IRLT measurements in each retinal section was taken as the mean (mIRLT) value.

\section{Hyperchromatic INL nuclei}

The INL nuclei were studied independently by two masked examiners (AWS and GGO) using light microscopy with oil immersion $(1250 \times)$ at three randomised areas of each retina. The cells were examined for the presence of condensed nuclear chromatin. The data collected from both examiners were pooled. The average numbers of hyperchromatic (HC) nuclei within the INL were recorded.

\section{Apoptosis of ganglion cells}

Apoptotic ganglion cells were morphologically detected and counted under light microscopy $(550 \times)$ using a terminal deoxynucleotid transferase assay in situ. Ten regions per retina were randomly sampled for apoptotic ganglion cells using a computerised image analysis program (analySIS 2.0).

\section{RESULTS}

On day 1, the mean SNP treated mIRLT was 203.3 (SEM 18.1) $\mu \mathrm{m}$ compared with a mean thickness of 145.4 (7.4) $\mu \mathrm{m}$ in the control retina (fig 1 ). The difference is statistically significant (two way ANOVA; $p=0.004$ ). Melatonin injection resulted in similar mIRLT values between the SNP treated retina and the controls ( $t$ test; $\mathrm{p}=0.228$ ). The mIRLT of the untreated controls (142.4 (7.8) $\mu \mathrm{m})$ was not significantly different from the saline treated retina ( $t$ test; $\mathrm{p}=0.68$ ).

On day 4, SNP also had a significant effect (two way ANOVA; $p=0.003$ ) on the mIRLT (fig 2 ). In the control rats, the mIRLT of the vehicle treated retina was comparable to the SNP treated retina ( $t$ test; $\mathrm{p}=0.172)$. In the melatonin treated rats, the mIRLT of the SNP treated retina was 206.3 (9.6) $\mu \mathrm{m}$, which was significantly thicker than 162.4 (6.6) $\mu \mathrm{m}$ of the vehicle treated eyes ( $t$ test; $p=0.006$ ). When the control and melatonin treated rats were compared, melatonin had no significant effects on mIRLT in the vehicle treated eyes ( $t$ test; $\mathrm{p}=0.432$ ) and the SNP injected eyes ( $t$ test; $\mathrm{p}=0.117)$.

Figures 3 and 4 depict the SNP induced INL cell changes. The data for all treatment groups are shown in table 1. No HC cells were detected in the control retina on day 1 and the data did not fit into the characteristic of Gaussian distribution. The numbers of animals that exhibited HC cells were thus analysed using a non-parametric paradigm. Intravitreal SNP injection significantly increased the HC counts after l day

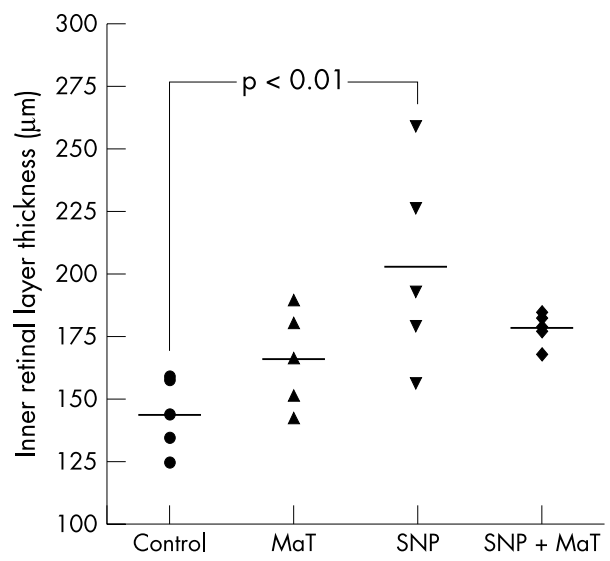

Figure 1 The inner retinal layer thickness 1 day after the treatments of sodium nitroprusside (SNP) and/or melatonin (MaT) injection $(n=5)$. The horizontal lines indicate the mean values of treatment groups.

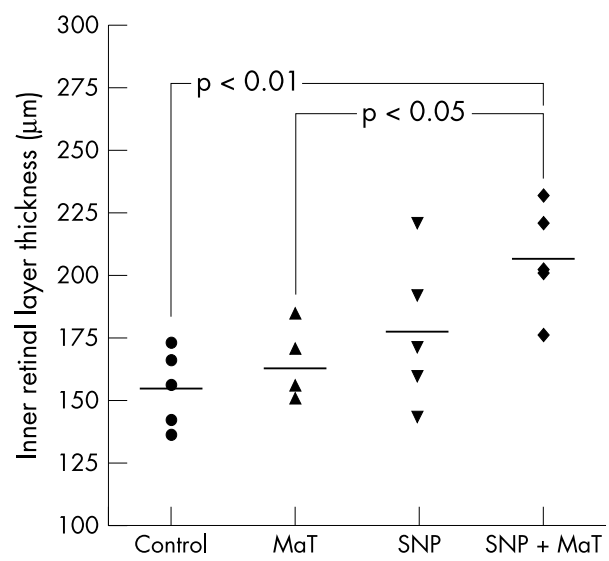

Figure 2 The inner retinal layer thickness 4 days after the treatments of sodium nitroprusside (SNP) and/or melatonin (MaT) injection $(n=5)$. The horizontal lines indicate the mean values of treatment groups.

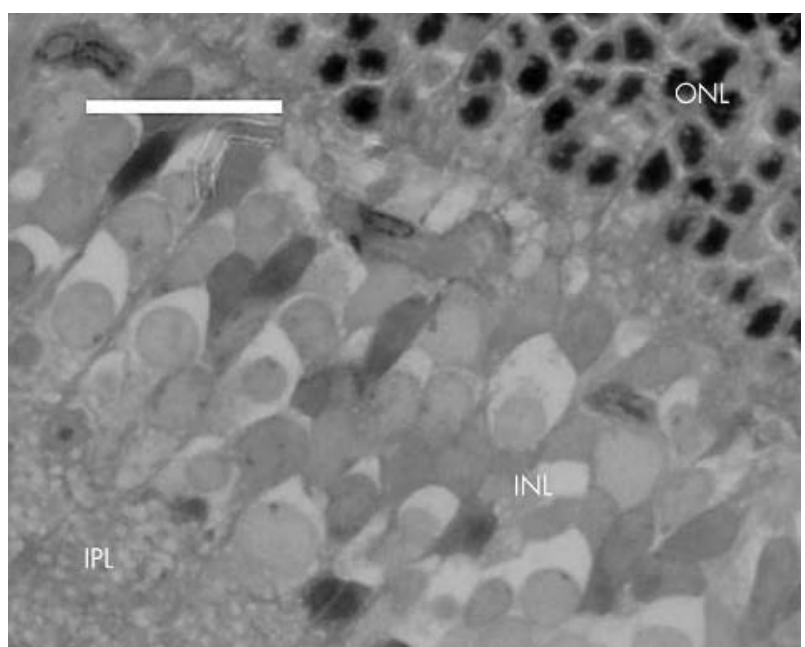

Figure 3 Microscopic cross section of the inner nuclear layer (INL) stained by toluidine blue: control. The figure also shows the outer nuclear layer (ONL) and inner plexiform layer (IPL). The scale bar represents a distance of $20 \mu \mathrm{m}$. (Magnification $\times 1250$ ).

$\left(\chi^{2} ; \mathrm{p}=0.010\right)$. Melatonin significantly reduced the $\mathrm{HC}$ counts $\left(\chi^{2} ; p=0.029\right)$ in the SNP injected eyes to a level comparable to the vehicle injected eyes. On day 4 , the HC counts were also significantly increased by the SNP treatment $\left(\chi^{2} ; p=0.038\right)$ but melatonin injection resulted in the same number of HC samples compared with controls $\left(\chi^{2} ; p=1.0\right)$.

Similar findings had been obtained from the ganglion cells apoptotic assay (fig 5). The number of apoptotic cells increased significantly 1 day after the SNP injection (Newman-Keuls, $\mathrm{p}<0.05)$. Four days afterwards, the mean number of apoptotic nuclei maintained at a significantly higher value (Newman-Keuls, $\mathrm{p}<0.05$ ). On the other hand, melatonin injection before the intravitreal SNP treatment reduced the expression of apoptotic ganglion cells. Melatonin reduced the SNP induced apoptotic ganglion cell counts from 5.0 (1.0) to $3.0(0.5)$ (mean (SEM)) for day 1 and from 8.0 (1.0) to $5.0(0.5)$ for day 4. Both differences were found to be statistically significant (Newman-Keuls, $\mathrm{p}<0.05$ ).

\section{DISCUSSION}

As a nitrovasodilator, NO has been proposed as a therapeutic agent for glaucoma ${ }^{13}{ }^{14}$ by virtue of its ability to modulate the retinal arterial tonus. ${ }^{15}$ Nevertheless, our data showed that 


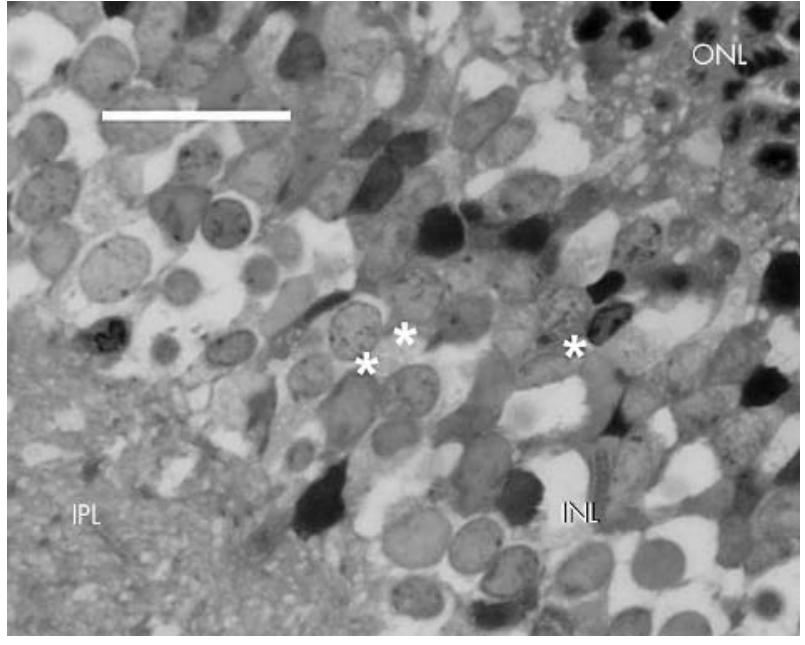

Figure 4 Microscopic cross section of the inner nuclear layer (INL) stained by tolvidine blue: sodium nitroprusside treatment. The figure also shows the outer nuclear layer (ONL) and inner plexiform layer (IPL). Cells with hyperchromatic condensation are marked (*). The scale bar represents a distance of $20 \mu \mathrm{m}$. (Magnification $\times 1250$ ).

excessive and uncontrolled intraocular NO elevation led to necrotic and apoptotic cell damage in the retina-retinal swelling, nuclear condensation, and apoptosis; responses very similar to those observed in $\mathrm{ONOO}^{-}$induced uveitis reported previously. ${ }^{16} \mathrm{NO}$ couples with superoxide anion radical to form $\mathrm{ONOO}^{-} \cdot{ }^{17} \mathrm{ONOO}^{-}$is a strong oxidant that devastates normal cellular functions-for example, decreases cell membrane fluidity, increases DNA fragmentation, ${ }^{18}$ depletes ATP, and disrupts mitochondria. ${ }^{19}$ Our data demonstrated an acute effect of NO on the cells leading to transient retinal oedema. This oedema subsided but the nuclear damage sustained in the following 4 days. These results demonstrated an apparent damaging effect of NO on the retina and the question as to whether therapeutic application of NO would further compromise the viability of surviving retinal cells in the glaucoma patient becomes critical. ${ }^{20}$

Ko $e t a^{21}$ showed that a non-specific antioxidant enhanced the protective effect of neurotrophic factor against retinal ganglion cells death. Melatonin is an antioxidant that potentially preserves a number of physiological functions. ${ }^{9}$ In our study, administering melatonin intraperitoneally before the intravitreal injection of SNP protected the retina from NO toxicity in the first 24 hours. This observation is

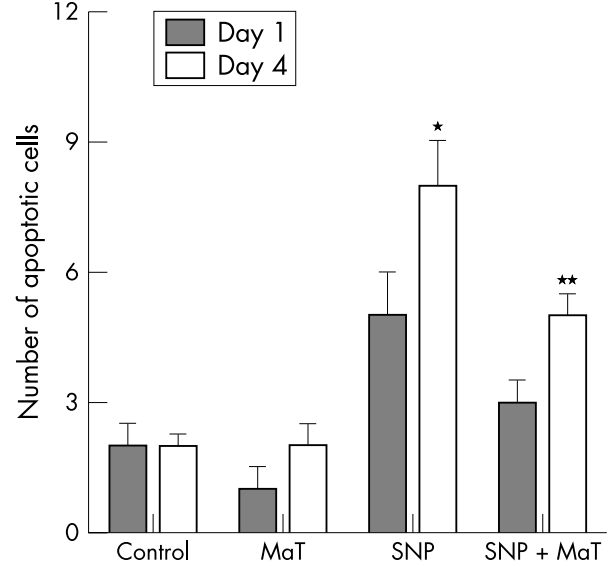

Figure 5 Apoptosis in the retinal ganglion cells $(n=5)$ after the treatments of sodium nitroprusside (SNP) and/or melatonin (MaT). ${ }^{*} p<0.05$ versus all conditions; ${ }^{* *} p<0.05$ versus all controls.

consistent with earlier data reported on the protection of melatonin in acute inflammation. ${ }^{22}$ This protective effect was not apparent in the present study, however, after 4 days. It is likely that $\mathrm{ONOO}^{-}$might have induced a self propagating chain reaction of oxidative damage in the retina. The secondary generation of alkoxyl and peroxyl radicals at cellular level ${ }^{23}$ is a time dependent process which may have been responsible for the prolonged oxidative damage. It is also possible that melatonin has been metabolised leading to restricted anti oxidative effects.

Melatonin may have protected the retina from NO induced damage by scavenging NO directly, ${ }^{24}$ or indirectly through its derivative $\mathrm{ONOO}^{-}{ }^{22}$ In this reaction, melatonin is oxidised to form the melatonyl radical cation. ${ }^{25}$ Subsequently, melatonin undergoes nitrosation and oxidation to form 1-nitrosomelatonin and 1-hydroxymelatonin. ${ }^{26}$ These melatonin derivatives have virtually no known toxicity in humans ${ }^{27}$ and its efficacy in reducing oxidative damage in the ocular structures-for example, retina ${ }^{28}{ }^{29}$ and lens, ${ }^{30}$ has been documented. Administration of melatonin (or other antioxidants) may have a role in protecting the retina against oxidative stressfor example, age related macular degeneration, ${ }^{31}$ glaucoma and uveitis. ${ }^{32}$ The current study further supports the role of melatonin as a protective agent against oxidative challenge. ${ }^{33}$ However, our data suggested that melatonin administration initially combated the oxidative challenge but probably not sufficient to arrest it completely. Although the dosage used in this protocol $(10 \mathrm{mg} / \mathrm{kg})$ was much higher than the daily

\begin{tabular}{|c|c|c|c|c|c|c|}
\hline & \multirow[b]{2}{*}{ Rat } & \multicolumn{2}{|l|}{ Vehicle } & \multirow[b]{2}{*}{ Rat } & \multicolumn{2}{|l|}{ MaT } \\
\hline & & Saline & SNP & & Saline & SNP \\
\hline \multirow{6}{*}{ Day 1} & 1 & 0 & 1.5 & 11 & 0 & 0 \\
\hline & 2 & 0 & 4.4 & 12 & 0.3 & 0 \\
\hline & 3 & 0 & 4.4 & 13 & 0 & 0 \\
\hline & 4 & 0 & 0 & 14 & 0 & 0.8 \\
\hline & 5 & 0 & 0.6 & 15 & 0 & 0 \\
\hline & Mean (SD) & $0(0)$ & $2.17(2.06)$ & Mean (SD) & $0.06(0.12)$ & $0.17(0.37)$ \\
\hline \multirow[t]{6}{*}{ Day 4} & 6 & 1.9 & 4.4 & 16 & 0 & 4.2 \\
\hline & 7 & 0 & 4.1 & 17 & 1.5 & 2.7 \\
\hline & 8 & 0 & 4.5 & 18 & 0 & 1.2 \\
\hline & 9 & 0 & 3.8 & 19 & 0.4 & 3.2 \\
\hline & 10 & 0.1 & 0.4 & 20 & 0 & 4.4 \\
\hline & Mean (SD) & $0.39(0.42)$ & $3.44(1.74)$ & Mean (SD) & $0.37(0.64)$ & $3.13(1.27)$ \\
\hline
\end{tabular}


level of melatonin supplement taken by human $(\sim 0.1 \mathrm{mg} /$ $\mathrm{kg}$ ), a discontinued supply of melatonin in this experimental protocol could account for the partial protection. More data will be required to characterise the bioavailability of melatonin to the retina. The clinical application of antioxidants in retinal protection deserves further investigation.

In conclusion, NO significantly increased the mIRLT, nuclear condensation of INL cells, and apoptotic ganglion cells in the first day. After 4 days, the swelling response subsided but the number, of HC nuclei and apoptotic ganglion cells were still greater than the normal levels. Previous administration of melatonin protected the retina from NO induced cellular changes within the first day but the effect did not last over 4 days.

\section{ACKNOWLEDGEMENTS}

The study was supported by The Hong Kong Polytechnic University Research Grants 351, 352, G-T485. AWS was a visiting scholar at the Centro de Investigacion Biomedica de Occidente (IMSS), Mexico.

\section{Authors' affiliations}

A W Siu, G G Ortiz, Division de Neurociencias, Centro de Investigacion Biomedica de Occidente (IMSS), Guadalajara, Jalisco, Mexico A W Siv, C H To, Laboratory of Experimental Optometry, Department of Optometry and Radiography, The Hong Kong Polytechnic University, Hong Kong, China

G G Ortiz, R J Reiter, Department of Cellular and Structural Biology, Mail Code 7762, University of Texas Health Science Center at San Antonio, San Antonio, TX 78229-3900, USA

G Benitez-King, Instituto Nacional de Psiquiatria, Departamento de Neurofarmacologia, DIC, Lorenzo Huipulco, Mexico

Correspondence to: A W Siv, PhD, Department of Optometry and Radiography, The Hong Kong Polytechnic University, Kowloon, HKSAR, China; ORSIUA@inet.polyu.edu.hk

Accepted for publication 3 January 2004

\section{REFERENCES}

1 Siu AW, Leung MCP, To $\mathrm{CH}$, et al. Total retinal nitric oxide production is increased in intraocular pressure-elevated rats. Exp Eye Res 2002;75:401-6.

2 Beckman JS, Beckman TW, Chen J, et al. Apparent hydroxyl radical production by peroxynitrite: implications for endothelial injury from nitric oxide and superoxide. Proc Natl Acad Sci USA 1990;87:1620-4.

3 Pigeolet E, Remacle J. Susceptibility of glutathione peroxidase to proteolysis after oxidative alteration by peroxides and hydroxyl radicals. Free Radic Biol Med 1991;11:191-5.

4 Garcia JJ, Reiter RJ, Guerrero JM, et al. Melatonin prevents changes in microsomal membrane fluidity during induced lipid peroxidation. FEBS Lett 1997; 408:297-300

5 Edward DP, Lam TT, Shahinfar S, et al. Amelioration of light-induced retinal degeneration by a calcium overload blocker. Flunarizine. Arch Ophthalmol 1991; 109:554-62.

6 Siu AW, To $\mathrm{CH}$. Nitric oxide and hydroxyl radical-induced retinal lipid peroxidation in vitro. Clin Exp Optom 2002;85:378-82.
7 Meyer P, Pache M, Loeffler KU, et al. Melatonin MT-1-receptor immunoreactivity in the human eye. Br J Ophthalmol 2002;86:1053-7.

8 Reiter RJ. Pineal melatonin: cell biology of its synthesis and of its physiological interactions. Endocr Rev 1991;12:151-80.

9 Reiter RJ, Tan DX, Cabrera J, et al. The oxidant/antioxidant network: role of melatonin. Biol Signals Recept 1999;8:56-63.

10 Reiter RJ, Guerrero JM, Escames G, et al. Prophylactic actions of melatonin in oxidative neurotoxicity. Ann NY Acad Sci 1997;825:70-8.

11 Siu AW, Reiter RJ, To CH. Pineal indoleamines and vitamin E reduce nitric oxide-induced lipid peroxidation in rat retinal homogenates. J Pineal Res 1999;27:122-8.

12 Cazevieille C, Osborne NN. Retinal neurones containing kainate receptors are influenced by exogenous kainate and ischaemia while neurones lacking these receptors are not-melatonin counteracts the effects of ischaemia and kainate. Brain Res 1997;755:91-100.

13 Nathanson JA. Nitrovasodilators as a new class of ocular hypotensive agents. J Pharmacol Exp Ther 1992;260:956-65.

14 Schuman JS, Erickson K, Nathanson JA. Nitrovasodilator effects on intraocular pressure and oufflow facility in monkeys. Exp Eye Res 1994;58:99-105.

15 Delaey C, Van de Voorde J. Retinal arterial tone is controlled by a retinalderived relaxing factor. Circ Res 1998;83:714-20.

16 Wu GS, Zhang J, Rao NA. Peroxynitrite and oxidative damage in experimental autoimmune uveitis. Invest Ophthalmol Vis Sci 1997;38:1333-9.

17 Beckman JS, Crow JP. Pathological implications of nitric oxide, superoxide and peroxynitrite formation. Biochem Soc Trans 1993;21:330-4.

18 Salgo MG, Squadrito GL, Pryor WA. Peroxynitrite causes apoptosis in rat thymocytes. Biochem Biophys Res Commun 1995;215:1111-8.

19 Murphy MP. Nitric oxide and cell death. Biochim Biophys Acta 1999;1411:401-14.

20 Becquet F, Courtois $Y$, Goureau $O$. Nitric oxide in the eye: multifaceted roles and diverse outcomes. Surv Ophthalmol 1997:42:71-82.

21 Ko ML, Hu DN, Ritch R, et al. The combined effect of brain-derived neurotrophic factor and a free radical scavenger in experimental glaucoma. Invest Ophthalmol Vis Sci 2000;41:2967-71.

22 Cuzzocrea S, Zingarelli B, Gilad E, et al. Protective effect of melatonin in carrageenan-induced models of local inflammation: relationship to its inhibitory effect on nitric oxide production and its peroxynitrite scavenging activity. J Pineal Res 1997;23:106-16.

23 Marnett $\mathrm{L}$, Wilcox AL. The chemistry of lipid alkoxyl radicals and their role in metal- amplified lipid peroxidation. Biochem Soc Symp 1995;61:65-72.

24 Noda Y, Mori A, Liburdy R, et al. Melatonin and its precursors scavenge nitric oxide. J Pineal Res 1999;27:159-63.

25 Zhang H, Squadrito GL, Pryor WA. The reaction of melatonin with peroxynitrite: formation of melatonin radical cation and absence of stable nitrated products. Biochem Biophys Res Commun 1998;251:83-7.

26 Blanchard B, Pompon D, Ducrocq C. Nitrosation of melatonin by nitric oxide and peroxynitrite. J Pineal Res 2000;29:184-92.

27 Nordlund JJ, Lerner AB. The effects of oral melatonin on skin color and on the release of pituitary hormones. J Clin Endocrinol Metab 1977;45:768-74.

28 Siv AW, Reiter RJ, To CH. The efficacy of vitamin E and melatonin as antioxidants against lipid peroxidation in rat retinal homogenates. J Pineal Res 1998;24:239-44.

29 Marchiafava PL, Longoni B. Melatonin as an antioxidant in retinal photoreceptors. J Pineal Res 1999;26:184-9.

30 Li ZR, Reiter RJ, Fujimori O, et al. Cataractogenesis and lipid peroxidation in newborn rats treated with buthionine sulfoximine: preventive actions of melatonin. J Pineal Res 1997;22:117-23

31 Liang FQ, Godley BF. Oxidative stress-induced mitochondrial DNA damage in human retinal pigment epithelial cells: a possible mechanism for RPE aging and age-related macular degeneration. Exp Eye Res 2003;76:397-403.

32 Bashir S, Harris G, Denman MA, et al. Oxidative DNA damage and cellular sensitivity to oxidative stress in human autoimmune diseases. Ann Rheum Dis 1993;52:659-66.

33 Reiter RJ. Melatonin: clinical relevance. Best Pract Res Clin Endocrinol Metab 2003; 17:273-85. 